\title{
Economic response that can be achieved from including genomic information to the terminal sire index of beef cattle
}

C Duthie, R Sawalha, E Navajas, R Roehe, T Roughsedge

Scottish Agricultural College, Edinburgh, United Kingdom

Email: carol-anne.duthie@sac.ac.uk

Introduction Genomic selection (GS) utilises information about the association of large numbers of SNP markers located throughout the genome with phenotypic information. This has become feasible due to the availability of large numbers of SNP markers and the development of the bovine SNP chip. Selection index methods can be utilized for deterministic modelling of the potential benefits of including genomic information in genetic improvement programmes. The aim of this study was to investigate the benefits of applying genomic selection to the terminal sire index of beef cattle considering training population size and different breeding structures.

Materials and methods Selection index theory was applied to investigate the response of the beef terminal sire index under conventional selection or GS given the structure of the UK beef industry. Breeding objectives of the terminal sire index incorporate carcass weight, carcass fat score, carcass conformation score, gestation length and calving difficulty. Currently recorded traits include birth weight, weight at 200 and 400 days, muscle score, muscle and fat depth, gestation length and calving difficulty. Parameters were obtained from UK beef genetic evaluations and information for the breeding goal traits were obtained from Amer et al. (1998). Selection strategies were derived from the structure of the beef industry which was calculated from UK beef genetic evaluations data (Table 1). The effect of different breeding structures was investigated. This included selection based on young sires, where no progeny information is available, and selection based on older sires. Genomic information was included in the selection index model based on the theory of Dekkers et al. (2007). Accuracies of GEBVs are predicted based on trait heritability, number of phenotyped animals in the training population, the number of QTL underlying the trait and the effective population size (Ne) (Daetwyler et al. 2008; Goddard 2009). In this research the effect of different training population sizes (1000 to 5000) and the effect of Ne of 100 and 500 were investigate.

Table 1 Selection strategies based on the structure of the UK beef industry.

\begin{tabular}{lllll}
\hline \hline & \multicolumn{2}{l}{$\begin{array}{l}\text { Proportion } \\
\text { candidates } \\
\text { selected }\end{array}$} & \multicolumn{3}{l}{ of } & \multicolumn{2}{l}{$\begin{array}{l}\text { Generation } \\
\text { Interval }\end{array}$} \\
& Sires & Dams & Sires & Dams \\
\hline $\begin{array}{l}\text { Young } \\
\text { sires }\end{array}$ & 0.06 & 0.53 & 3.00 & 6.25 \\
$\begin{array}{l}\text { Industry } \\
\text { average }\end{array}$ & 0.04 & 0.53 & 4.55 & 6.25 \\
\hline \hline
\end{tabular}

Results The results of selection with and without GS are shown in Table 2. Under conventional selection, the economic response is similar between the two breeding structures, however the slightly higher response $(5 \%)$ when selecting older sires is due to higher accuracy of breeding values due to the availability of phenotypic records on more relatives. Including genomic information increases the economic response to selection in both breeding structures, however the magnitude of the response in comparison to no GS is higher when selecting younger sires (up to 33\% increase) in comparison to the industry average (up to $21 \%$ increase) where selection is based on approximately $40 \%$ young sires (generation interval $<3$ ) and $60 \%$ of older sires. The size of the training population influences the economic response that can be achieved when including genomic information, where the highest response is achieved with a training population of 5000. However, this is constrained by the breeding program structure and Ne. Larger training population sizes had more impact when younger sires were selected. Furthermore, the rate of economic response was higher for Ne of 100 than 500 .

Conclusions The results show that there is potential benefit that can be achieved from including genomic information in selection programmes in beef cattle. The benefit that can be achieved is highest when selecting younger sires compared to older sires. This research outlines the importance of the training population size and $\mathrm{Ne}$ as these constrain the potential benefit that can be achieved. GS is expected to be of particular benefit for traits which have low heritability and are difficult to measure or are only available late in the animals life or are sex limited. Therefore, GS may facilitate the inclusion of further traits, such as residual feed intake, in breeding programmes which are important to the efficiency of beef cattle.

Acknowledgements The authors gratefully acknowledge funding from the Scottish Government and DEFRA. We would also like to acknowledge Peter Amer (Abacus Biotech, New Zealand) for providing the selection index program.

\section{References}

Amer, P.R., Crump, R., and Simm, G. 1996. Animal Science 67, 445-454.

Daetwyler, H.D., Villanueva, B. and Woolliam, J.A. 2008. Plos One.10, 1-8.

Dekkers, J.C.M. 2007. Journal of Animal Breeding and Genetics 124, 331-341.

Goddard, M.E. 2009. Genetica 136, 245-25 